\title{
Intra- and Inter-Investigator Reliability of Measurement of Lip-Seal Strength in Adults
}

\author{
Takayuki Ueda, Takeshi Oki, Midori Ohta, Koichiro Ogami and \\ Kaoru Sakurai \\ Department of Removable Prosthodontics \& Gerodontology, Tokyo Dental College, \\ 2-9-18 Kanda-Misakicho, Chiyoda-ku, Tokyo 101-0061, Japan
}

Received 6 June, 2018/Accepted for Publication 10 July, 2018

Published Online in J-STAGE 15 March, 2019

\begin{abstract}
The purpose of this methodological study was to clarify intra- and inter-investigator reliability of lip-seal strength measurement in adults. Lip-seal strength was measured with a digital medical strain gauge using the button-pull method. The coefficient of variation (CV) of intra-investigator reliability of measurement in 24 healthy adult volunteers (16 men, 8 women; mean age, $26 \pm 3$ years) ranged from 0.088 to 0.103 (mean for 3 investigators, 0.098). The intraclass correlation coefficient (ICC $[1,1]$ ) of intra-investigator reliability of this method of lip-seal strength measurement ranged from 0.759 to 0.832 (mean, 0.804). The ICC $(2,1)$ of inter-investigator reliability of the measured values was 0.737. The CV of intra-investigator reliability of lip-seal strength measurement by a single investigator in 54 adult patients (14 men, 40 women; mean age, $49 \pm 16$ years) was 0.072 . The ICC $(1,1)$ of intra-investigator reliability of this measurement method of lip-seal strength was 0.863 . A CV of approximately 0.2 or lower is considered to indicate excellent agreement; an ICC of approximately 0.75 or higher is considered indicative of good reliability. These results demonstrate that measurement of lip-seal strength in adults with this device offers sufficient intra- and inter-investigator reliability.
\end{abstract}

Key words: Oral function — Lip - Methodology - Measurement reliability — Adults

\section{Introduction}

The lips have a number of important functions commencing from birth in the act of suckling, and some studies have reported a relationship between lip-seal and malalignment during the growth period ${ }^{1,13)}$. The lips also play roles in speech, food ingestion, and swallowing in adults and elderly individuals.
These functions include bilabial articulation (such as in production of the $/ \mathrm{m} /$ sound), introduction of food into the oral cavity and subsequent bolus formation, and generation of adequate swallowing pressure. Elderly individuals presenting with choking on food due to impairment of swallowing function during the pharyngeal stage were reported to show significantly poorer labial pressure and move- 
ment ${ }^{15)}$. This suggests that lip-closing function also plays a role during the pharyngeal stage of feeding and swallowing. A reduction was observed in the motor function of the lips in elderly individuals with mild cognitive impairment ${ }^{17}$. An association was demonstrated between not only tongue strength, but also lip force and sarcopenia in elderly individuals during the post-acute phase of illness ${ }^{12)}$. Reduced maximum restraining lip force was reported to be a quantifiable symptom of impairment following stroke ${ }^{14)}$. Taken together, these results indicate the importance of accurately evaluating lip function in adults and elderly individuals.

There are two important aspects of muscular function of the tongue and lips: muscle strength and motor function (dexterity). It has been noted that measurement of oral diadochokinesis is useful in evaluating motor function of the tongue and lips ${ }^{18)}$. A probetype tongue pressure gauge ${ }^{4,16)}$ or pressure sensor sheet ${ }^{5,9)}$ are commonly used to measure tongue pressure in determining muscle strength. The muscle strength of the lips has been called lip-seal ${ }^{1)}$, lip-strength ${ }^{13)}$, vertical labial pressure ${ }^{15)}$, lip-closing strength ${ }^{11)}$, lipforce ${ }^{3,12)}$, lip-closing force ${ }^{6)}$, maximum voluntary lip-closing force ${ }^{7)}$, and maximum restraining lip-force ${ }^{14)}$; and various methods have been developed for its evaluation.

One study reported measurement of lipclosing strength using the previously established button-pull method ${ }^{2}$. In this method, a digital force gauge is used to measure the maximum force required to pull a button retained by the lips. A measurement device based on this concept has been used in pediatric dentistry and is now applied in clinical practice. One study used this method to measure lip-closing strength in 554 children aged 3 to 12 years and calculated standard values according to age $\mathrm{e}^{11}$.

However, the reliability of this method in adults remains to be investigated. The purpose of the present study was to evaluate intraand inter-investigator reliability in measurement of lip-seal strength measurement with this device. We believe that establishing such

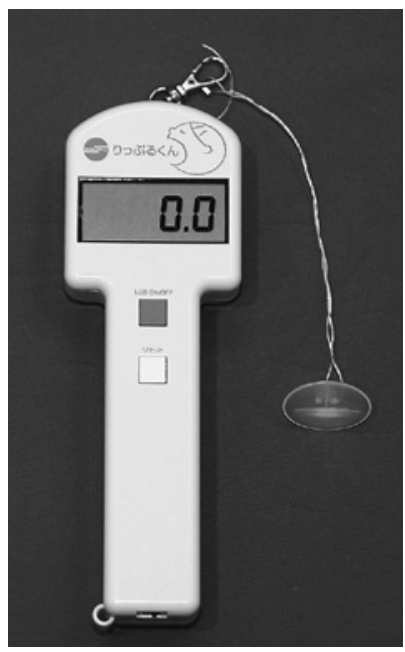

Fig. 1 Digital medical strain gauge for measurement of lip-seal strength (Lipplekun)

reliability would be of significance for future studies focused on investigating oral function and treatment in elderly patients, especially those requiring accurate evaluation of lip function, such as in individuals with oral hypofunction and/or cerebrovascular disease.

\section{Materials and Methods}

Lip-seal strength was measured with a digital medical strain gauge (Lipplekun; Shofu, Kyoto, Japan), in accordance with a method reported previously (Fig. 1) ${ }^{11}$. The Lipple button (Shofu) for measurement (Fig. 2) was attached to the tip of this device with $0.2 \mathrm{~m}$ of dental floss (Johnson \& Johnson, New Brunswick, NJ, USA). During measurement, the participants were required to sit on a chair with the head positioned such that the Frankfurt plane was parallel to the floor (Fig. 3). They were then instructed to tighten their lips to resist the exerted pulling force, but not apply negative pressure in the oral cavity, move the head or body, or tip the head forward.

The investigator stood in front of the participant, placed the Lipple button between 


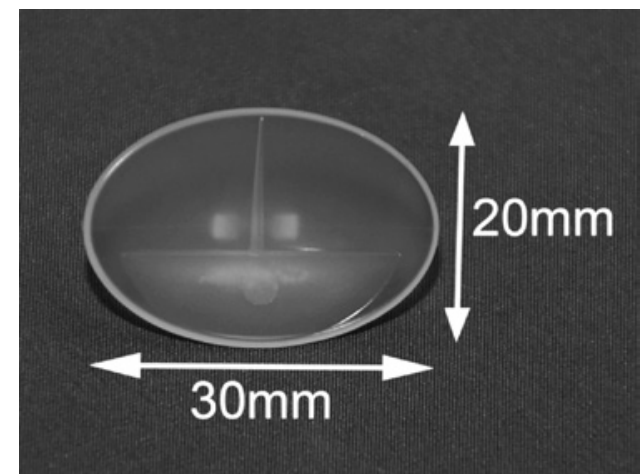

Fig. 2 Button used in button-pull measurement (Lipple button). Button was attached to Lipplekun

the participant's teeth and lips, confirmed that the button was located at the center of the dentition, and instructed them to close their lips firmly. The measurement device was positioned horizontal to the floor so that an indicator light shone onto the participant's subnasale at the midline of the face. The button was then slowly pulled for 3 seconds, with the measurement device held at this position until the button was completely pulled from the lips. The maximum force $(\mathrm{N})$ during pulling was regarded as the lip-seal strength. Each participant was allowed to practice this procedure several times before measurement. Actual measurement was performed after confirming that the participant fully understood the measurement method. Measurement in each participant was performed 3 times with sufficient rest intervals. The protocol of this study was approved by the Ethics Committee of Tokyo Dental College (approval no. 755).

\section{Intra- and inter-investigator reliability in volunteer participants}

Twenty-four students at Tokyo Dental College (16 men, 8 women; mean age, $26 \pm 3$ years) participated in this portion of the study. Three dentists (experience as dentist, 2 to 3 years) who were not familiar with this method of measurement were chosen from outside of our team. They were then trained in taking

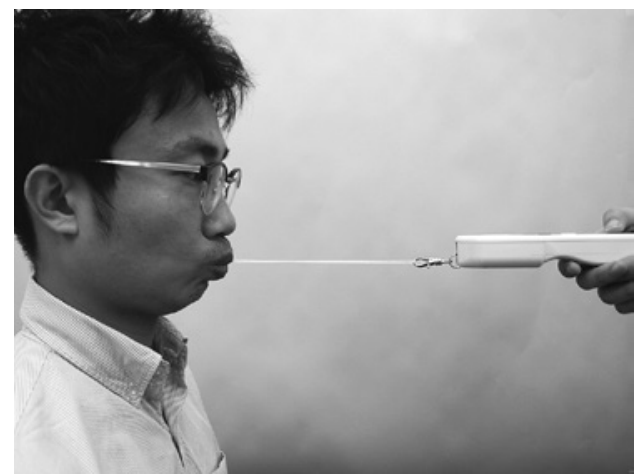

Fig. 3 Measurement of lip-seal strength

the measurements to be obtained prior to commencing the procedure. During the actual experiment, each dentist was required to measure lip-seal strength 3 times in each participant. These 3 measurements were performed continuously based on the normal clinical protocol of this method. This procedure was repeated by all 3 dentists in each participant within one day. An interval sufficient to prevent muscle fatigue was provided between each session with one of the dentists. The order in which the dentists were selected to perform measurements was chosen at random, and none were informed of the values obtained by any of the other investigators.

\section{Intra-investigator reliability in patients}

Fifty-four outpatients aged 20 years or older with no acute systemic or oral cavity symptoms (14 men, 40 women; mean age, $49 \pm 16$ years) participated in this portion of the study. Most of the participants were visiting the hospital for a periodic dental checkup. One registered nurse, who was familiar with clinical physiological examinations and this method, performed the measurements. For a comparative control, the same investigator measured the maximum tongue pressure 3 times in each participant with a JMS tongue-pressure measurement device (JMS, Hiroshima, Japan).

\section{Statistical analysis}

Reliability was evaluated with the intraclass 
Table 1 Coefficient of variation of intra-investigator reliability in lip-seal strength measurements made by 3 investigators

\begin{tabular}{lccccc}
\hline \hline & $\mathrm{n}$ & \multicolumn{4}{c}{ Coefficient of variation } \\
\cline { 3 - 6 } & & Minimum & Maximum & Mean & $\mathrm{SD}$ \\
\hline Investigator 1 & 24 & 0.020 & 0.330 & 0.101 & 0.079 \\
Investigator 2 & 24 & 0.020 & 0.340 & 0.088 & 0.073 \\
Investigator 3 & 24 & 0.000 & 0.300 & 0.103 & 0.077 \\
\hline
\end{tabular}

$\mathrm{SD}$, standard deviation

Table 2 Intraclass correlation coefficient (ICC) $(1,1)$ of intra-investigator reliability in lip-seal strength measurements made by 3 investigators

\begin{tabular}{|c|c|c|c|c|c|c|c|c|}
\hline & \multirow{2}{*}{$\mathrm{n}$} & \multirow{2}{*}{$\begin{array}{c}\text { ICC } \\
(1,1)\end{array}$} & \multicolumn{2}{|c|}{$\begin{array}{l}95 \% \text { Confidence } \\
\text { Interval }\end{array}$} & \multicolumn{4}{|c|}{ F test } \\
\hline & & & Lower & Upper & Value & df1 & df2 & $p$ value \\
\hline Investigator 1 & 24 & 0.821 & 0.686 & 0.911 & 14.803 & 23 & 48 & 0.000 \\
\hline Investigator 2 & 24 & 0.832 & 0.703 & 0.917 & 15.850 & 23 & 48 & 0.000 \\
\hline Investigator 3 & 24 & 0.759 & 0.591 & 0.877 & 10.442 & 23 & 48 & 0.000 \\
\hline
\end{tabular}

correlation coefficient (ICC) and coefficient of variation $(\mathrm{CV})$. To assess intra-investigator reliability, values were measured 3 times by each investigator and the ICC $(1,1)$ and CV calculated. To assess inter-investigator reliability, the ICC $(2,1)$ and CV were calculated using the maximum values of the 3 measurements obtained by each investigator. The Pearson's correlation coefficient between lipseal strength and maximum tongue pressure was calculated. The significance level was set at 0.05 . Statistical analysis was performed with SPSS Statistics 24 (IBM Corporation, Armonk, NY, USA).

\section{Results}

The CV for intra-investigator reliability of lip-seal strength measurement obtained by the 3 investigators in the 24 volunteer participants ranged from 0.088 to 0.103 (mean for the 3 investigators, 0.098; Table 1). The ICC
$(1,1)$ of this method ranged from 0.759 to 0.832 (mean, 0.804; Table 2). The ICC $(2,1)$ for inter-investigator reliability of the measured values was 0.737 (Table 3 ).

The CV values for lip-seal strength and maximum tongue pressure measured by a single investigator in 54 patients are shown in Table 4; the ICC $(1,1)$ values of this method are shown in Table 5. Mean lip-seal strength was $12.3 \pm 3.2 \mathrm{~N}$, and mean maximum tongue pressure was $36.0 \pm 7.3 \mathrm{kPa}$ (Table 6). Pearson's correlation coefficient between lip-seal strength and maximum tongue pressure was $0.314(\mathrm{p}=0.021)$ (Fig. 4).

\section{Discussion}

Several methods have been proposed for evaluating the reliability of tests of physiological function, but none has yet been accepted as the standard. Two items were evaluated in the present study: intra- and inter-investigator 
Table 3 Intraclass correlation coefficient (ICC) $(2,1)$ of inter-investigator reliability in measurements of lip-seal strength (maximum value) made by 3 investigators

\begin{tabular}{|c|c|c|c|c|c|c|c|c|}
\hline & \multirow{2}{*}{$\mathrm{n}$} & \multirow{2}{*}{$\begin{array}{l}\text { ICC } \\
(2,1)\end{array}$} & \multicolumn{2}{|c|}{$\begin{array}{l}95 \% \text { Confidence } \\
\text { Interval }\end{array}$} & \multicolumn{4}{|c|}{$\mathrm{F}$ test } \\
\hline & & & Lower & Upper & Value & df1 & df2 & $p$ value \\
\hline Investigators $1-3$ & 24 & 0.737 & 0.541 & 0.868 & 11.032 & 23 & 46 & 0.000 \\
\hline
\end{tabular}

Table 4 Coefficient of variation of intra-investigator reliability in values measured 3 times by single investigator

\begin{tabular}{lccccc}
\hline \hline & & \multicolumn{4}{c}{ Coefficient of variation } \\
\cline { 3 - 6 } & & Minimum & Maximum & Mean & SD \\
\hline Lip-Seal Strength & 54 & 0.009 & 0.253 & 0.072 & 0.046 \\
Tongue Pressure & 54 & 0.011 & 0.229 & 0.077 & 0.057 \\
\hline
\end{tabular}

SD, standard deviation

Table 5 Intraclass correlation coefficient (ICC) $(1,1)$ of intra-investigator reliability in values measured 3 times by single investigator

\begin{tabular}{|c|c|c|c|c|c|c|c|c|}
\hline & \multirow{2}{*}{$\mathrm{n}$} & \multirow{2}{*}{$\begin{array}{c}\text { ICC } \\
(1,1)\end{array}$} & \multicolumn{2}{|c|}{$\begin{array}{l}95 \% \text { Confidence } \\
\text { Interval }\end{array}$} & \multicolumn{4}{|c|}{$\mathrm{F}$ test } \\
\hline & & & Lower & Upper & Value & df1 & df2 & $p$ value \\
\hline Lip-Seal Strength & 54 & 0.863 & 0.795 & 0.913 & 19.866 & 53 & 108 & 0.000 \\
\hline Tongue Pressure & 54 & 0.715 & 0.597 & 0.812 & 8.543 & 53 & 108 & 0.000 \\
\hline
\end{tabular}

reliability. The CV and ICC, which are widely used to evaluate reliability, were also employed. The CV for intra-investigator reliability was 0.098 for the volunteer participants and 0.072 for the patients. Although the CV represents variation in test values, there is no clear judgment criterion. According to the judgment criteria for kappa values used for a similar purpose $^{10)}$, a CV of 0.2 or lower is considered to represent excellent agreement, and one of 0.2 to 0.4 substantial agreement. The CV value found in the present study shows that the variation in repeated measurements in a single participant was small enough to indicate and that the method used was reliable.

The ICC $(1,1)$ for intra-investigator reliability was 0.804 for the volunteer participants and 0.863 for the patients. The ICC $(2,1)$ for inter-investigator reliability was 0.737 . Although there are no clear judgment criteria for ICC values, a value of approximately 0.75 or higher is considered indicative of good reliability $^{10}$. One earlier study investigating the reliability of lip-seal strength measurement using a lip-force meter reported that the ICC $(1,1)$ and ICC $(2,1)$ of intra- and inter-investigator reliability were 0.83 and 0.71 , respectively ${ }^{3)}$. The present results indicate that the reliability of the method used here was com- 
Table 6 Means and standard deviations of lipseal strength and maximum tongue pressure in patients

\begin{tabular}{lccc}
\hline \hline & $\mathrm{n}$ & Mean & $\mathrm{SD}$ \\
\hline Lip-Seal Strength (N) & 54 & 12.3 & 3.2 \\
Tongue Pressure (kPa) & 54 & 36.0 & 7.3 \\
\hline
\end{tabular}

parable with that seen in that previous report.

One of the most widely used tests of oral function seeks to determine muscular strength through measurement of tongue pressure. In the present study, the $\mathrm{CV}$ and ICC $(1,1)$ indicated greater intra-investigator reliability for lip-strength measurement than for tongue-pressure measurement. The mean \pm SD for lip-seal strength in the patients was $12.3 \pm 3.2 \mathrm{~N}$, while the $\mathrm{CV}$ was 0.26 . Thus, the intra- and inter-investigator concordance rates with this measurement method were lower than the variation in lip-seal strength among the participants. An earlier study found that the $\mathrm{CV}$ of labial pressure during ingestion was $0.26 \pm 0.14$ in healthy young adults, $0.34 \pm 0.17$ in healthy elderly individuals, and $0.34 \pm 0.19$ in elderly individuals living in a nursing care facility ${ }^{15}$. The CV obtained in the present study was smaller than the physiological variation in lip-seal strength that would be observed during ingestion of food, suggesting that this measurement method is sufficiently reliable to be applied both in a clinical and research setting.

There are several possible reasons for the high reliability of this measurement method. First, the procedure is simple because it was originally developed for use in children. And because the device has a simple structure and can be used easily, it may also be suitable for measurement in elderly patients and those with cerebrovascular disease. In addition, positioning is always accurate because the device is equipped with an indicator light. The button is pulled while the guide light illuminates the subnasale; therefore, an accurate positional relationship between the face

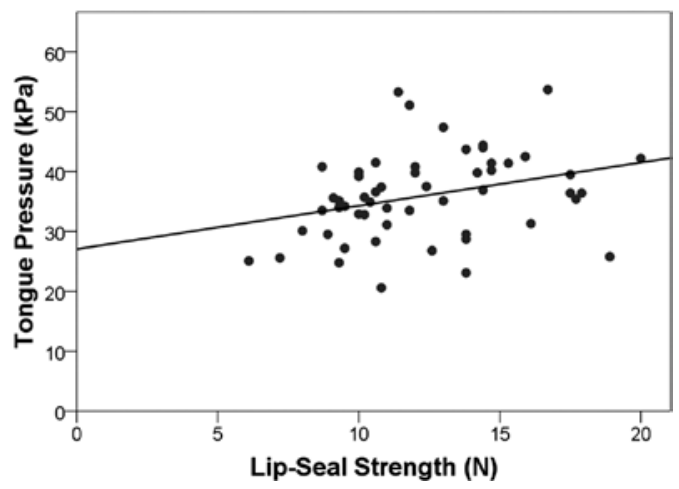

Fig. 4 Scatter plots of lip-seal strength and maximum tongue pressure $(\mathrm{n}=54)(\mathrm{y}=27.06+0.72 \mathrm{x})$

Significant correlation was noted between two parameters.

and the measurement device is maintained during pulling, which reduces variation in measurement.

In investigating inter-investigator reliability, the maximum value among the 3 measurements obtained was considered as representative in each participant. The mean or median of the measured values may also be used as representative values. Generally, when the ICC is calculated using the mean, the resulting value is high. This indicates that use of the maximum value is suitable for evaluation of maximum muscular strength. Although the measured value may decrease due to measurement-induced muscle fatigue and measurement errors, it is unlikely to increase due to error. This is another reason the maximum value was adopted here. However, it is important to know that if the button is pulled very quickly during measurement of lip-seal strength, the value may be abnormally high.

Oral function involves numerous complex components, so a combination of several tests is necessary for a thorough evaluation. According to the Japanese Society of Gerodontology, reduced function in 3 or more of 7 evaluation items, including tongue pressure, occlusal force, and motor function of the tongue and lips, indicates a diagnosis of oral hypofunction $^{8)}$. When oral hypofunction 
is diagnosed, more detailed function tests are necessary to prepare a management plan. The results of the present study clarified the reliability of the present method of lip-seal strength measurement in adults. This method may contribute to studies on oral function and treatment in elderly patients, especially those requiring accurate evaluation of lip function, such as in individuals with oral hypofunction and/or cerebrovascular disease.

In conclusion, the present results indicate that measurement of lip-seal strength in adults with the present device offers good reliable intra- and inter-investigator reliability.

\section{Disclosure}

The authors declare no conflict of interest related to this study.

\section{References}

1) Fränkel R (1980) Lip seal training in the treatment of skeletal open bite. Eur J Orthod 2: 219-228.

2) Fukami A, Saitoh I, Inada E, Oku T, Iwase Y, Takemoto Y, Yamada C, Iwasaki T, Hasegawa H, Kubota N, Murakami T, Harada K, Nishi M, Kinjo S, Igata N, Hayasaki H, Yamasaki Y (2014) A reproducibility method to test lipclosing strength in preschool children. Cranio 28:232-237.

3) Hägg M, Olgarsson M, Anniko M (2008) Reliable lip force measurement in healthy controls and in patients with stroke: a methodologic study. Dysphagia 23:291-296.

4) Hayashi R, Tsuga K, Hosokawa R, Yoshida M, Sato Y, Akagawa Y (2002) A novel handy probe for tongue pressure measurement. Int J Prosthodont 15:385-388.

5) Hori K, Ono T, Tamine K, Kondoh J, Hamanaka S, Maeda Y, Dong J, Hatsuda M (2009) Newly developed sensor sheet for measuring tongue pressure in swallowing. J Prosthodont Res 53:28-32.

6) Jung MH, Yang WS, Nahm DS (2003) Effects of upper lip closing force on craniofacial structures. Am J Orthod Dentofacial Orthop 123: 58-63.
7) Kaede K, Kato T, Yamaguchi M, Nakamura N, Yamada K, Masuda Y (2016) Effects of lipclosing training on maximum voluntary lipclosing force during lip pursing in healthy young adults. J Oral Rehabil 43:169-175.

8) Minakuchi S, Tsuga K, Ikebe K, Ueda T, Tamura F, Nagao K, Furuya J, Matsuo K, Yamamoto K, Kanazawa M, Watanabe Y, Hirano H, Kikutani T, Sakurai K (2018) Oral hypofunction in the older population: position paper of the Japanese Society of Gerodontology in 2016. Gerodontology (in press) doi: $10.1111 /$ ger.12347

9) Ono T, Hori K, Nokubi T (2004) Pattern of tongue pressure on hard palate during swallowing. Dysphagia 19:259-264.

10) Portney LG, Watkins MP (2009) Statistical measures of reliability, Foundations of Clinical Research: Applications to Practice, 3rd ed., pp.585-618, Pearson/Prentice Hall, Upper Saddle River, New Jersey.

11) Saitoh I, Inada E, Kaihara Y, Nogami Y, Murakami D, Ishitani N, Sawami T, Iwase Y, Nakajima T, Kubota N, Sakurai K, Tsujii T, Shirazawa Y, Hanasaki M, Kurosawa M, Goto M, Nosou M, Kozai K, Yamasaki Y, Hayasaki H (2017) The relationship between lip-closing strength and the related factors in a cross-sectional study. Pediatr Dent J 27:115-120.

12) Sakai K, Nakayama E, Tohara $H$, Takahashi $O$, Ohnishi S, Tsuzuki H, Hayata M, Takehisa T, Takehisa Y, Ueda K (2018) Diagnostic accuracy of lip force and tongue strength for sarcopenic dysphagia in older inpatients: a crosssectional observational study. Clin Nutr (Epub ahead of print) doi: 10.1016/j.clnu.2018.01. 016

13) Satomi M (2001) The relationship of lip strength and lip sealing in MFT. Int J Orofacial Myology 27:18-23.

14) Schimmel M, Leemann B, Schnider A, Herrmann FR, Kiliaridis S, Müller F (2013) Changes in oro-facial function and hand-grip strength during a 2-year observation period after stroke. Clin Oral Investig 17:867-876.

15) Tamura F, Fukui T, Kikutani T, Machida R, Yoshida M, Yoneyama T, Hamura A (2009) Lip-closing function of elderly people during ingestion: comparison with young adults. Int J Orofacial Myology 35:33-43.

16) Tsuga K, Yoshikawa M, Oue H, Okazaki Y, Tsuchioka H, Maruyama M, Yoshida M, Akagawa Y (2012) Maximal voluntary tongue pressure is decreased in Japanese frail elderly persons. Gerodontology 29:e1078-e1085.

17) Watanabe $Y$, Hirano $H$, Arai H, Morishita $S$, Ohara Y, Edahiro A, Murakami M, Shimada H, Kikutani T, Suzuki T (2017) Relationship 
between frailty and oral function in community-dwelling elderly adults. J Am Geriatr Soc 65:66-76.

18) Yamada A, Kanazawa M, Komagamine Y, Minakuchi S (2015) Association between tongue and lip functions and masticatory performance in young dentate adults. J Oral Rehabil 42:833-839.

\section{Correspondence:}

Dr. Takayuki Ueda

Department of Removable Prosthodontics \& Gerodontology,

Tokyo Dental College,

2-9-18 Kanda-Misakicho, Chiyoda-ku,

Tokyo 101-0061, Japan

Tel: + 81-3-6380-9201

E-mail: uedat@tdc.ac.jp 